\title{
Constellation Design of The Beidou Satellite Navigation System and Performance Simulation
}

\author{
Wang Xiaomeng \\ Civil Aviation University of China \\ School of Electronic and Information Engineering \\ Tianjin, 13752416223 \\ wangxiaomeng.399@163.com
}

\author{
Liu Ruihua \\ Civil Aviation University of China \\ School of Electronic and Information Engineering \\ Tianjin, 13034362088 \\ rhliu_cauc@163.com
}

\begin{abstract}
To improve the positioning accuracy and reliability of Beidou , an analysis of the Beidou satellite navigation system has been performed; combination of technical experience of the foreign global satellite navigation system and the current release of technical information, we have designed Beidou satellite navigation system constellation, by satellite visibility analysis and a variety of DOP's numerical comparison of the different design options, we can prove the Beidou second-generation constellation program can meet the needs of the global satellite location, especially in China and its surrounding areas, the designed Beidou second-generation satellite navigation system have more precise positioning compared to other global navigaiton system.
\end{abstract}

Keywords- Beidou satellite navigation system; satellite constellation design; the DOP value; simulation

\section{INTRODUCTION}

Beidou satellite navigation system is a new all-day-lasting and regional satellite navigation positioning system, which is developed by China own, provides fast positioning to users and novel short digital message communications and timing services. Now the space segment of Beidou satellite navigation system which China is building consists of five geostationary satellites and 30 non-geostationary satellites, it will provide two services: open service and authorization service. China plans to cover the Asia-Pacific region by "Beidou" system in about 2012, and to cover global coverage in about 2020. China is implementing the construction of the Beidou satellite navigation system, has successfully launched 13 Beidou navigation satellites. There is no doubt that the Beidou system will play a major role in the military and civilian region. The article mainly plan constellation of the 35 satellites for the Beidou system to ensure optimal positioning.

\section{BEIDOU CONSTELLATION DESIGN}

\section{A. Satellite Orbital Theory Introduction}

Considering the period of earth autobiography movement is 24 hours, the following four kinds of tracks are commonly used in the navigation satellite:

(1)The geostationary orbit (GEO)

The satellite orbital plane coincides with the equatorial plane, running a period of 24 hours, stationary above the

Funded projects: basic scientific research and operational requirements of the Central Universities Civil Aviation University of China ZXH2010B004 equator, the orbit is a circular orbit in the geocentric inertial coordinate system.

(2)Medium earth orbit (MEO)

Height parameters of the satellite is generally 10,000 to $25,000 \mathrm{~km}$, a maximum length of time that the satellite appears in the ground plane is for a few hours. The application of this track to establish a global communications satellite system only needs 2-3 orbital planes to complete the global coverage.

(3) Inclined geosynchronous orbit (IGSO)

The return period is one sidereal day (1436.1 minutes). The center located at a set of longitude on the equator , the altitude is same as geostationary satellites, IGSO is also an efficient regional constellation between GEO and MEO.

\section{(4) Huge elliptical orbit (HEO)}

This is a large eccentricity orbit with its apogee over the high latitudes of the northern hemisphere, their nadir trajectory moves slowly, it can maintain the effective running of 10 hours for 5 days. The altitude of this kind of satellites change obviously, it is very detrimental for the design of the channel.

\section{B. Geometric Errors}

Constellation positioning (Dilution Of Precision, DOP) is an important factor to influence the positioning accuracy, it's also an important indicator to measure the merits of a navigation satellite constellation.

Set pseudorange equation (1).

$$
\rho_{i}=\sqrt{\left(x-x_{i}\right)^{2}+\left(y-y_{i}\right)^{2}+\left(z-z_{i}\right)^{2}}+C t_{u}
$$

$(x, y, z)$ refers user receiver position, $\left(x_{i}, y_{i}, z_{i}\right)$ refers the position of the satellite $\mathrm{i}, \rho_{i}$ refers the pseudorange measurements from the user to the satellite, $C$ refers radio wave propagation speed, $t_{u}$ refers the clock interval between the user receiver clock and Beidou system clock. So

$$
\left[\begin{array}{l}
\Delta \rho_{1} \\
\Delta \rho_{2} \\
\vdots \\
\Delta \rho_{i}
\end{array}\right]=\left[\begin{array}{cccc}
\frac{\partial \rho_{1}}{\partial x} & \frac{\partial \rho_{1}}{\partial y} & \frac{\partial \rho_{1}}{\partial z} & 1 \\
\frac{\partial \rho_{2}}{\partial x} & \frac{\partial \rho_{2}}{\partial y} & \frac{\partial \rho_{2}}{\partial z} & 1 \\
\vdots & \vdots & \vdots & \vdots \\
\frac{\partial \rho_{i}}{\partial x} & \frac{\partial \rho_{i}}{\partial y} & \frac{\partial \rho_{i}}{\partial z} & 1
\end{array}\right]\left[\begin{array}{c}
\Delta x \\
\Delta y \\
\Delta z \\
C \Delta t_{u}
\end{array}\right]=\mathbf{H} \Delta \mathbf{X}
$$


When $i=4$

$$
\Delta \mathbf{X}=\mathbf{H}^{-1} \Delta \boldsymbol{\rho}
$$

When $i>4$, we can get the least squares

$$
\begin{aligned}
\boldsymbol{\Delta} \mathbf{X} & =\left(\mathbf{H}^{\mathrm{T}} \mathbf{H}\right)^{-1} \mathbf{H}^{\mathrm{T}} \boldsymbol{\Delta} \boldsymbol{\rho} \\
d \mathbf{X} & =\left(\mathbf{H}^{\mathrm{T}} \mathbf{H}\right)^{-1} \mathbf{H}^{\mathrm{T}} d \boldsymbol{\rho}
\end{aligned}
$$

We can get (6) based on the definition of covariance

$$
\begin{aligned}
\operatorname{cov}(d \mathbf{X}) & =E\left[d \mathbf{X} d \mathbf{X}^{\mathrm{T}}\right] \\
& =E\left[\left(\mathbf{H}^{\mathrm{T}} \mathbf{H}\right)^{-1} \mathbf{H}^{\mathrm{T}} d \boldsymbol{\rho} d \boldsymbol{\rho}^{\mathrm{T}} \mathbf{H}\left(\mathbf{H}^{\mathrm{T}} \mathbf{H}\right)^{-1}\right] \\
& =\left(\mathbf{H}^{\mathrm{T}} \mathbf{H}\right)^{-1} \mathbf{H}^{\mathrm{T}} \operatorname{cov}(d \boldsymbol{\rho}) \mathbf{H}\left(\mathbf{H}^{\mathrm{T}} \mathbf{H}\right)^{-1}
\end{aligned}
$$

Generally speaking, the distribution of $d \boldsymbol{\rho}$ 's each component assumed identical and mutually independent, its variance is equal to the satellite pseudoranges squared error factor $\sigma_{U E R E}$. Then

$$
\operatorname{cov}(d \boldsymbol{\rho})=\mathbf{I}_{n \times n} \sigma_{U E R E}^{2}
$$

Put (7) into equation (6), available

$$
\operatorname{cov}(d \mathbf{X})=\left(\mathbf{H}^{\mathrm{T}} \mathbf{H}\right)^{-1} \sigma_{U E R E}^{2}
$$

Under these assumptions, the error covariance between the calculated position and the actual deviation is exactly the scalar product of the matrix $\left(\mathbf{H}^{\mathrm{T}} \mathbf{H}\right)^{-1}$. The component of matrix $\left(\mathbf{H}^{\mathrm{T}} \mathbf{H}\right)^{-1}$ quantitative refered that how to transform pseudorange error into every component of $d \mathbf{X}$ 's covariance .

Based on definition, the accuracy factor DOP parameters is obtained the division between sum of components of $\operatorname{cov}(d \mathbf{X})$ and $\sigma_{\text {UERE }}$. The most general parameter called the GDOP (Geometrical Dilution Of Precision)

$$
G D O P=\frac{\sqrt{\sigma_{x_{u}}^{2}+\sigma_{y_{u}}^{2}+\sigma_{z_{u}}^{2}+\sigma_{C_{b}}^{2}}}{\sigma_{U E R E}}
$$

The GDOP relationship is given with a component in the form of $\left(\mathbf{H}^{\mathrm{T}} \mathbf{H}\right)^{-1}$, this component form

$$
\left(\mathbf{H}^{\mathrm{T}} \mathbf{H}\right)^{-1}=\left[\begin{array}{cccc}
D_{11} & D_{12} & D_{13} & D_{14} \\
D_{21} & D_{22} & D_{23} & D_{24} \\
D_{31} & D_{32} & D_{33} & D_{34} \\
D_{41} & D_{42} & D_{43} & D_{44}
\end{array}\right]
$$

GDOP can be calculated out as the square root of the matrix trace $\left(\mathbf{H}^{\mathrm{T}} \mathbf{H}\right)^{-1}$

$$
G D O P=\sqrt{D_{11}+D_{22}+D_{33}+D_{44}}
$$

GDOP is the geometric factor, which represents the amount of amplification from the standard deviation of the measurement error to the solution. By the formula (11) we can get that GDOP is merely a function of the geometric layout of the satellite / user.

It can be concluded the following characteristics of GDOP:

1) GDOP is the geometric magnification factor from measurement errors to position solver error. In the same observation precision $\sigma_{U E R E}$, the smaller of GDOP value, the higher positioning accuracy; otherwise lower. So it is essentially an error amplification factor.

2) GDOP is only related to the relative geometric layout between the user location and the positioning satellite, regardless of the chosen coordinate system.

There are other DOP parameters for the characterization of the position / time solution components which commonly used, for the position dilution of precision (PDOP), the horizontal dilution of precision (HDOP), the vertical dilution of precision (VDOP) and the time dilution of precision (TDOP), each component of $\left(\mathbf{H}^{\mathrm{T}} \mathbf{H}\right)^{-1}$ is used to represent as

$$
\begin{aligned}
& P D O P=\sqrt{D_{11}+D_{22}+D_{33}} \\
& H D O P=\sqrt{D_{11}+D_{22}} \\
& V D O P=\sqrt{D_{33}} \\
& T D O P=\sqrt{D_{44}}
\end{aligned}
$$

\section{Beidou Constellation Design}

At present, the world's major satellite navigation systems use the Walker Constellation layout. Walker Constellation consists of a group of circular orbit satellites, which run on the same orbital period and inclination, denoted by the Walker T/P/F. The satellites of each track are distributed evenly. The longitude of ascending node spacing between the raceway surfaces is also equally distribution,so $\mathrm{T}$ (number of satellites) $=\mathrm{s}$ (the satellite number of he same orbital plane) $\times \mathrm{P}$ (the number of orbital plane). The relative phase of satellite between two adjacent tracks is determined by the phase parameter F, F is the number of "gap" $\left(360^{\circ} / \mathrm{T}\right)$ between the most eastern satellite orbit and the most Western satellite orbit, it means that $\mathrm{F}$ is an integer from 0 to $\mathrm{P}-1$.

Satellite navigation need multiple coverage, more simultaneous visual satellites are needed in navigation application, for the selection of RNSS System constellation, we need to refer to foreign satellite navigation systems, the most widely used three foreign satellite navigation systems, are the U.S. Global Positioning System, GPS, and the former Soviet Union / Russian global navigation satellite system GLONASS and the EU's Galileo satellite navigation system GALILEO which is in building, according to data published by the three satellite navigation system, as well as long-term practice, available $\mathrm{e}^{[2 \sim 4]}$,

1) satellite constellation whose height less than $2000 \mathrm{~km}$ is inappropriate. The analysis showed that the number of satellites in 2000km highly Walker Constellation is four times than $20000 \mathrm{~km}$ highly walker constellation, which will make the system costs and maintenance costs soared.

2) In order to achieve the higher-level performance indicators, we need at least 24 satellites. The satellite altitude performance indicators is weakened with the increasing number of satellites. When the global constellation satellite number is greater than or equal to 27 , we don't need to consider the the satellite height's contribution for the accuracy.

3) In order to further improve the availability, we should increase the number of in-orbit backup satellite instead of constellation modification. 
According to information currently published ${ }^{[2 \sim 4]}$,the IGSO satellite can make full use of the advantages of GEO, as well as overcoming the weakness that its high latitude regions are always low elevation. IGSO has the same orbital altitude with GEO, its orbital inclination is greater than $0^{\circ}$.therefore it has the same orbital period as the Earth's rotation cycle, but its nadir trajectory on the ground in the equatorial symmetry axis "8"-shaped, the larger orbit inclination, the greater "8"-shaped region, and the greater coverage. For the future development of Chinese global satellite navigation system, in order to achieve greater coverage area navigation system, so that it can achieve the effect of satellite navigation systems in Europe and the United States, and to ensure more accurate positioning results in China and the surrounding areas, we choose to increase the number of IGSO satellites, to reach a compromise effect in constellation design.

Taking all above factors, the paper has designed 5GEO satellites +27MEO satellites constellation program, and the improved 5GEO satellites +27MEO satellite +3IGSO satellites constellation program, the number of 35 satellites is from No.1 to No.35, the five GEO satellite located at eastern longitude $58.75^{\circ}, 80^{\circ}, 110.5^{\circ}, 140^{\circ}, 160^{\circ}$, orbital altitude is $42378.137 \mathrm{~km}$, the orbit inclination angle is $0{ }^{\circ} ; 27 \mathrm{MEO}$ satellites are evenly distributed in three orbital planes, nine satellites in each plane, and the longitude of the three planes are respectively, $30^{\circ}, 150^{\circ}, 270^{\circ}$, the equally distribution of latitude from $0{ }^{\circ}$ to $320^{\circ}$ for 9 satellites, orbital altitude is $27878.137 \mathrm{~km}$, the orbital inclination angle is $55^{\circ}$; The locations of 3IGSO satellites are $\left(\mathrm{E} 240^{\circ}, \mathrm{N} 0^{\circ}\right),\left(\mathrm{E} 0^{\circ}, \mathrm{N} 120^{\circ}\right)$, $\left(\mathrm{E} 120^{\circ}, \mathrm{N} 240^{\circ}\right)$, the orbital altitude is $42378.137 \mathrm{~km}$ orbital inclination angle is $55^{\circ}$. Simulation of satellite visibility and DOP value on the the two constellation program will be shown.

\section{SATELLITE VISIBILITY SIMULATION AND CONTRAST}

\section{A. Satellite Visibility of the Same Program but Different Height Coverage Angle}

For 5GEO +27 MEO constellation design height coverage angle are $5^{\circ}, 10^{\circ}$, the observation time is for one hour, the observation point is Civil Aviation University of China (Latitude: N53.5 ${ }^{\circ}$; Longitude: E120.5 ${ }^{\circ}$ ), the satellite visibility of two different height coverage angles of are shown in Figure 1:
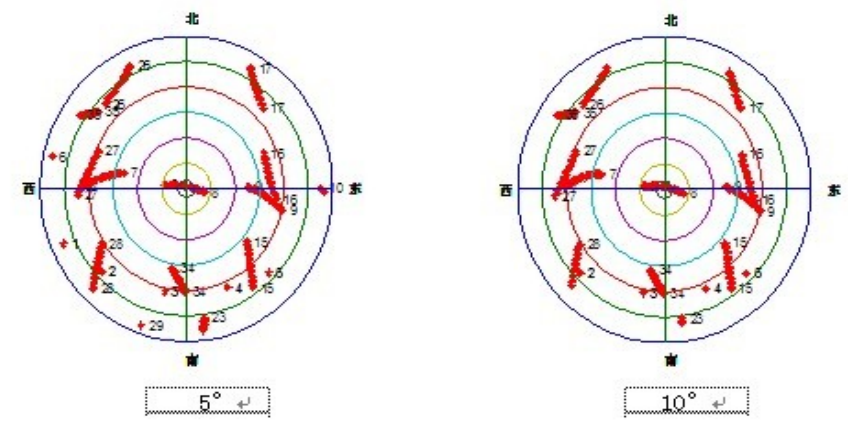

Fig.1 Visibility of satellite comparison chart by different satellite mask angle
The above two simulation pictures are from the sky plan view, the outermost circle is horizon $0^{\circ}$, and then inside one circle, increasing $15^{\circ}$. Numbers in Figure 1 refer to the satellite sequence number, the red line indicates the trajectory of the satellite, it can be clearly seen that the number of stars, and its trajectory become weak with increasing height of the coverage angle, that is satellite visibility becomes worse with the height coverage angle increasing. When height coverage angle is $5^{\circ}$, it not only can satisfy the requirement for fault monitoring and failure to exclude, and its satellite visibility is better than the case that the high coverage angle is $10^{\circ}$. Therefore, we set the height coverage angle to $5^{\circ}$ for the following simulation analysis.

Define abbreviations and acronyms the first time they are used in the text, even after they have been defined in the abstract. Abbreviations such as IEEE, SI, MKS, CGS, sc, dc, and rms do not have to be defined. Do not use abbreviations in the title or heads unless they are unavoidable.

\section{B. Satellite Visibility of the Same Height Coverage Angle but Different Program}

Simulation comparison of satellite visibility of the designed 5GEO +27 MEO program and 5GEO +27 MEO +3 IGSO program, the selected observation point is Civil Aviation University of China (Latitude: N53.5 ${ }^{\circ}$; Longitude: E120.5 ${ }^{\circ}$ ), the observation time is for one hour, the height coverage angle is $5^{\circ}$, the results are shown in Figure 2:

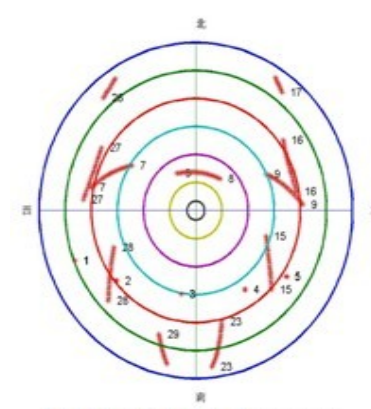

$5 \mathrm{GEO}+27 \mathrm{MEO}$

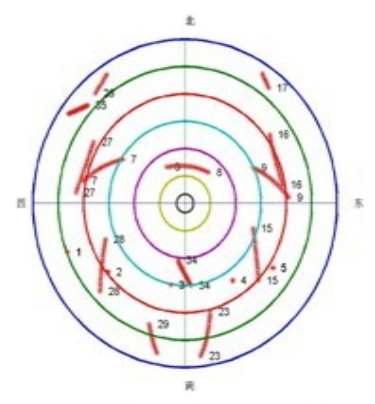

$5 \mathrm{GEO}+27 \mathrm{MEO}+3 \mathrm{IGSO}$
Fig.2 Visibility of satellite comparison chart by different satellite design

The Figure 2 shows that at the same observation point, the number 34 satellite and 35 satellite are visible 5GEO +27 $\mathrm{MEO}+3$ IGSO program, which are not visible in 5GEO +27 MEO program, so 5GEO+27MEO+3 IGSO program has better performance on satellite visibility analysis.

\section{SimUlATION AND COMPARISON OF DOP VALUES}

To test the positioning performance of the two Beidou satellite constellation programs designed.in China, we have chosen China's east, west,south and north boundary points to observe, the latitude and longitude of the four boundary point are: the most northern point ( $\mathrm{N} 53.5^{\circ}$, E120.5 ${ }^{\circ}$ ); the most southern point $\left(\mathrm{N} 4{ }^{\circ} \mathrm{E} 110^{\circ}\right)$; the most western point (N39 ${ }^{\circ}$, E73. $\left.{ }^{\circ}\right)$; the most eastern point $\left(\mathrm{N} 48^{\circ} \mathrm{E} 135^{\circ}\right)$, it can be concluded by the above four points' DOP value simulation analysis. 
A. Four Observation Points' DOP Value of 5GEO +27MEO Simulation Program

1) DOP simulation curve for 24 hours is shown in in Figure 3:
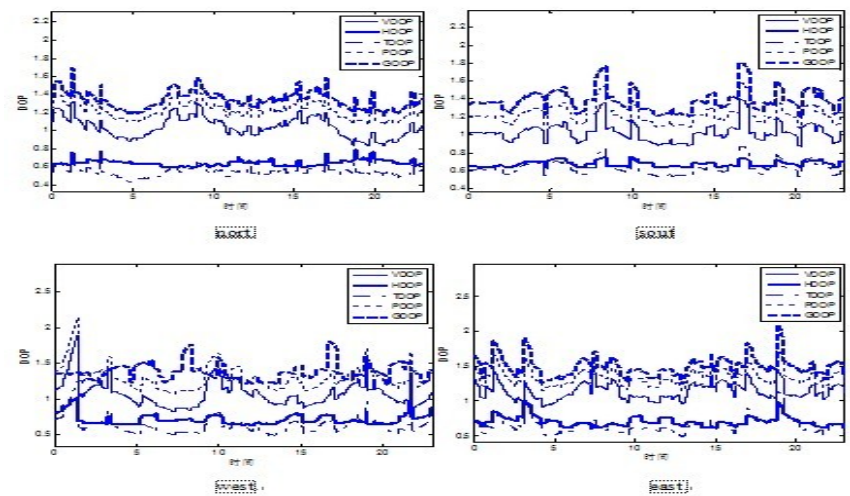

Fig.3 DOP simulation figure of 5GEO+27MEO program

2) the comparison of the above four points DOP value are shown in Table 1:

TAB. 1 DOP VALUES OF FOUR BOUNDARIES IN 5GEO+27MEO PROGRAM

\begin{tabular}{|c|c|c|c|c|c|}
\hline & & north & south & west & east \\
\hline $\max$ & \multirow{3}{*}{ §ీ } & 1.3182 & 1.3923 & 1.9082 & 1.4542 \\
\hline $\min$ & & 0.8223 & 0.8706 & 0.8183 & 0.9128 \\
\hline ave & & 1.0436 & 1.0257 & 1.0770 & 1.1360 \\
\hline $\max$ & \multirow{3}{*}{$\begin{array}{l}\text { ઠे } \\
\text { }\end{array}$} & 0.7882 & 0.7546 & 1.0227 & 1.0252 \\
\hline $\min$ & & 0.5921 & 0.6365 & 0.6246 & 0.6150 \\
\hline ave & & 0.6392 & 0.6743 & 0.7299 & 0.7094 \\
\hline $\max$ & \multirow{3}{*}{$\stackrel{\text { ڤे }}{\varrho}$} & 0.7406 & 0.8763 & 1.2465 & 1.1643 \\
\hline $\min$ & & 0.4449 & 0.5287 & 0.4800 & 0.4916 \\
\hline ave & & 0.5525 & 0.6327 & 0.6519 & 0.6443 \\
\hline $\max$ & \multirow{3}{*}{$\begin{array}{l}\text { ठิ } \\
\text { a. }\end{array}$} & 1.5184 & 1.5782 & 2.1650 & 1.7339 \\
\hline $\min$ & & 1.0603 & 1.0799 & 1.0708 & 1.1445 \\
\hline ave & & 1.2259 & 1.2280 & 1.3044 & 1.3394 \\
\hline $\max$ & \multirow{3}{*}{ 용 } & 1.6894 & 1.8052 & 2.4982 & 2.0855 \\
\hline $\min$ & & 1.1647 & 1.2024 & 0.5294 & 1.2456 \\
\hline ave & & 1.3450 & 1.3816 & 1.4591 & 1.4874 \\
\hline
\end{tabular}

B. Four Observation Points' DOP Value of 5GEO +27MEO +3IGSO Simulation Program

1) DOP simulation curve for 24 hours is shown in Figure 4 :

2) the comparison of the above four points DOP value are shown in Table 2:

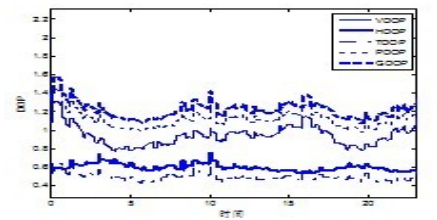

noof
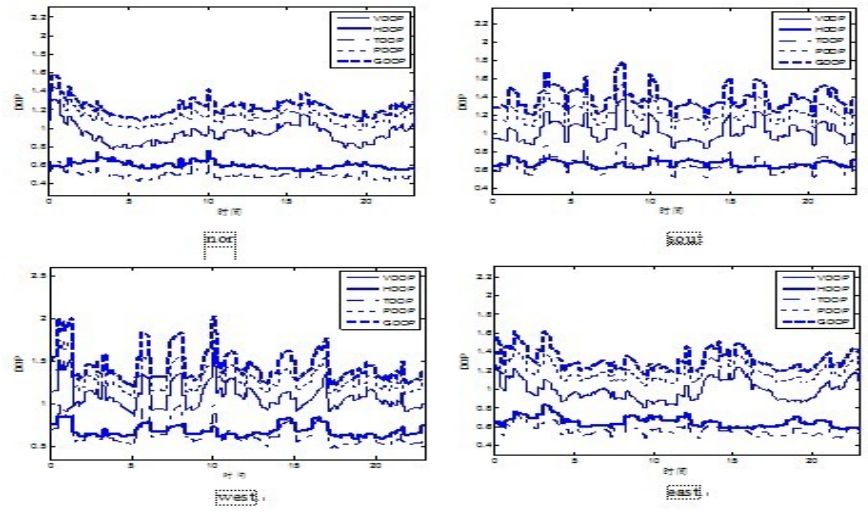

Fig.4 DOP simulation figure of 5GEO+27MEO+3IGSO program
TAB.2 DOP VALUES OF FOUR BOUNDARIES IN 5GEO+27MEO+3IGSO

\begin{tabular}{|c|c|c|c|c|c|}
\hline \multicolumn{6}{|c|}{ PROGRAM } \\
\hline & & north & south & west & east \\
\hline $\max$ & \multirow{3}{*}{ §ิ } & 1.3111 & 1.3734 & 1.6044 & 1.3199 \\
\hline $\min$ & & 0.7613 & 0.8288 & 0.8407 & 0.7906 \\
\hline ave & & 0.9472 & 1.0248 & 1.0948 & 0,9888 \\
\hline $\max$ & \multirow{3}{*}{ 悹 } & 0.7566 & 0.7660 & 0.8587 & 0.8385 \\
\hline $\min$ & & 0.5371 & 0.6125 & 0.5925 & 0.5638 \\
\hline ave & & 0.5984 & 0.6678 & 0.6896 & 0.6503 \\
\hline $\max$ & \multirow{3}{*}{ ڤి } & 0.6561 & 0.8941 & 0.9974 & 0.8235 \\
\hline $\min$ & & 0.4247 & 0.5229 & 0.4685 & 0.4551 \\
\hline ave & & 0.4972 & 0.6577 & 0.6493 & 0.5614 \\
\hline $\max$ & \multirow{3}{*}{ ڤิ } & 1.4376 & 1.5347 & 1.7708 & 1.4572 \\
\hline $\min$ & & 0.9720 & 1.0437 & 1.0552 & 1.0318 \\
\hline ave & & 1.1230 & 1.2244 & 1.2956 & 1.1867 \\
\hline $\max$ & \multirow{3}{*}{$\begin{array}{l}\text { ठे } \\
\text { 이 }\end{array}$} & 1.5802 & 1.7762 & 2.0323 & 1.6087 \\
\hline $\min$ & & 1.0675 & 1.1674 & 1.1683 & 1.1359 \\
\hline ave & & 1.2285 & 1.3901 & 1.4504 & 1.3134 \\
\hline
\end{tabular}

Figure 3, Figure 4 and Table 1, Table 2 of the 5 GEO +27 MEO constellation design and 5GEO+27MEO+3IGSO constellation design indicated that:

1)the maximum GDOP value is 1.69-2.50,1.58-2.03 range, the average GDOP value is 1.34-1.49,1.23-1.45 range, it can fully satisfy the requirements of all regions' navigation;

2)the DOP curves undulating, it indicates that the system continuity need to be improved;

3)the latter DOP values are less than the former, and the curve is relatively smaller, the latter DOP outperforms than the former.

\section{MOVING TARGET SATELLITE VISIBILITY AND DOP VALUE ANALYSIS}

In practical, there is little location for the stationary objects, and in most cases it requires accurate positioning system in the target movement, therefore, we choose a target movement trajectory for one day, then use the two design schemes to simulate the target's satellite visibility and DOP values.

The design trajectory of the target movement has been shown in Figure 5, the starting refered point in the geography (eastnorthup) coordinates is [000]:

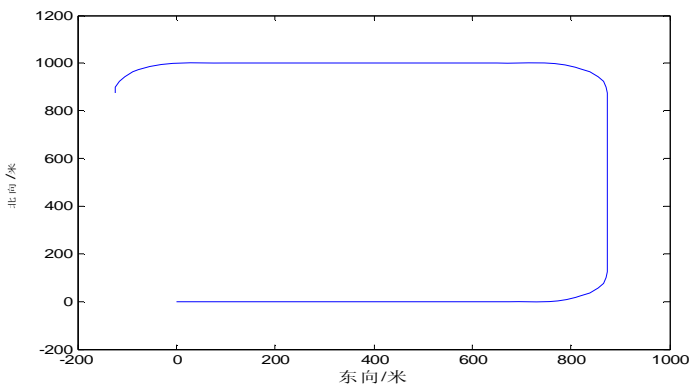

Fig.5 The target trajectory

The speed of the target in the three roads is $5 \mathrm{~m} / \mathrm{s}$, the delay is for $150 \mathrm{~s}$, the centripetal acceleration of the right-angle corner is $0.2 \mathrm{~m} / \mathrm{s}^{2}$. 


\section{A. Satellite Visibility Simulation of Two Different Programs} When the Target is Moving

The satellite visibility simulation of two different programs has been shown in Figure 6:

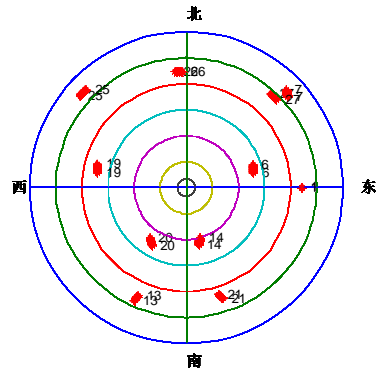

$5 \mathrm{GEO}+27 \mathrm{MEO}$

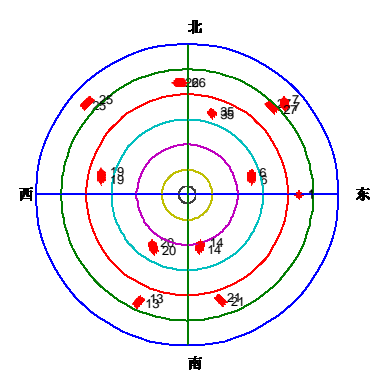

5GEO+27MEO+3IGSO
Fig.6 Satellite visibility simulation diagram of the moving target in two different programs

It can be seen by comparison of the two simulation graphs of satellite visibility that $5 \mathrm{GEO}+27 \mathrm{MEO}+3 \mathrm{IGSO}$ program can see No.35 satellite which is not seen in 5GEO+27 MEO program, and with the movement of the target, No.35 satellite has always been visible, so 5GEO +27MEO +3IGSO program's performance is superior.

\section{B. the DOP Value Comparison of Two Different Programs in When the Target is Moving}

The DOP value comparison of two different programs when the target is moving simulation has been shown in Table 3 :

TAB.3 THE DOP VALUE CONTRAST OF MOVING TARGET IN TWO DIFFERENT PROGRAMS

\begin{tabular}{|c|c|c|c|c|}
\hline Beidou constellation design & DOP & max & min & ave \\
\hline \multirow{4}{*}{5 GEO+27MEO } & VDOP & 1.4770 & 1.4389 & 1.4443 \\
\cline { 2 - 5 } & HDOP & 0.7673 & 0.7614 & 0.7644 \\
\cline { 2 - 5 } & TDOP & 0.7604 & 0.7582 & 0.7598 \\
\cline { 2 - 5 } & PDOP & 1.6354 & 1.6306 & 1.6341 \\
\cline { 2 - 5 } & GDOP & 1.8036 & 1.7983 & 1.8022 \\
\hline 5GEO+27MEO+3IGSO & VDOP & 1.3668 & 1.3471 & 1.3581 \\
\cline { 2 - 5 } & HDOP & 0.7350 & 0.7287 & 0.7318 \\
\cline { 2 - 5 } & TDOP & 0.7435 & 0.7380 & 0.7413 \\
\cline { 2 - 5 } & PDOP & 1.5489 & 1.5346 & 1.5427 \\
\cline { 2 - 5 } & GDOP & 1.7181 & 1.7028 & 1.7116 \\
\hline
\end{tabular}

It can be seen by the analysis and comparison of the DOP value of the moving target, improved 5GEO+27MEO +3IGSO constellation design scheme's five DOP values are smaller than another scheme, therefore, in the positioning of the moving target, 5GEO+27MEO+3IGSO constellation design has more advantages.

\section{CONCLUSION}

It can be seen by the analysis of the second-generation Beidou satellite constellation that the constellation design for the Beidou satellite navigation system has good coverage quality in China and its nearby areas, and it has equally accurate positioning of the moving target, while improving 5GEO+27MEO+3IGSO constellation design has an advantage over another program, but it still need related improvements on the continuity aspect.

Beidou navigation system can only be used in positioning in China and its surrounding area, and the performance has some shortcomings, but it is established in accordance with the needs of China's national conditions, the structure of multisystem which is compatible shared with other navigation systems will be formed in the near future. Therefore, no matter that the Beidou system is a primary navigation system, a single navigation system or an assisted navigation system, the study of the Beidou satellite navigation system is of great significance .

\section{REFERENCES}

[1] Xu Qifeng regional satellite navigation system satellite constellation [J] Surveying Engineering, 2001, 10 (1): 1-5.

[2] Paul Massatt, Micheal Zeitzew. The GPS Constellation DesignCurrent and Projected[C]. Proceedings of The National Technical Meeting "NAVIGATION 2000". Long Beach. California, 1998, 569574.

[3] Chu Hai-bin, Zhang Nai-tong, Gu Xue-mai. New Regional Satellite Positioning Constellation Scheme Discussion[J]. Journal of Beijing Institute of Technology, 2005, 14 (4) : 471-476.

[4] M. Rothaeher. Orbits of Satellite System in space Geodesy. Schweizerischen Geodatischen Commissions Volume46. 1993.

[5] Liu Genyou, Hao Xiaoguang, Chen Xiaofeng. Discussion of our second-generation satellite navigation system coverage northward expansion [J]. Geodesy and Geodynamics, 2007, 27(5): 115-118.

[6] Dai Hongfa. Discussion on the development and application of the Beidou navigation system [J]., 2010, （19） : 54-55.

[7] Liu Jiyu, The new development of GNSS Global Navigation Satellite System [J], remote sensing and control, 2007, 28 (4) : 14-15.

[8] Tan Shusen. Development and thinking of Beidou satellite navigation system [J]. Astronautics, 2008, 29(2): 392-396.

[9] Introduction and Application Prospects of the second generation Beidou satellite navigation system [J]. Satellite and network, 2008, 11.

[10] Kai T. Chinese Navigation Localization Satellite System Progress[J]. Aerospace China, Issue8. 2002. 